\title{
In-process 3D geometry reconstruction of objects produced by direct light projection
}

\author{
Andersen, Ulrik Vølcker; Pedersen, David Bue; Hansen, Hans Nørgaard; Nielsen, Jakob Skov
}

Published in:

International Journal of Advanced Manufacturing Technology

Link to article, DOI:

$10.1007 / \mathrm{s} 00170-013-4778-3$

Publication date:

2013

Link back to DTU Orbit

Citation (APA):

Andersen, U. V., Pedersen, D. B., Hansen, H. N., \& Nielsen, J. S. (2013). In-process 3D geometry reconstruction of objects produced by direct light projection. International Journal of Advanced Manufacturing Technology, 68(1-4), 565-573. https://doi.org/10.1007/s00170-013-4778-3

\section{General rights}

Copyright and moral rights for the publications made accessible in the public portal are retained by the authors and/or other copyright owners and it is a condition of accessing publications that users recognise and abide by the legal requirements associated with these rights.

- Users may download and print one copy of any publication from the public portal for the purpose of private study or research.

- You may not further distribute the material or use it for any profit-making activity or commercial gain

- You may freely distribute the URL identifying the publication in the public portal 


\title{
In-process 3D Geometry Reconstruction of Objects Produced by Direct Light Projection
}

\author{
Ulrik Vølcker Andersen • David Bue Pedersen • Hans Nørgaard Hansen • \\ Jakob Skov Nielsen
}

Received: date / Accepted: date

\begin{abstract}
Additive Manufacturing (AM) allows closeto unrestrained geometrical freedom in part design. The ability to manufacture geometries of such complexity is however limited by the difficulty of verifying the tolerances of these parts. Tolerances of features that are inaccessible with traditional measuring equipment such as coordinate measuring machines cannot be verified easily. This problem is addressed by developing an inline reverse engineering and 3D reconstruction method that allows a true to scale reconstruction of a part being additively manufactured. In earlier works $[1,2]$ this method has shown its potential with 3D Printing (3DP) and Selective Laser Sintering (SLS) additive manufacturing processes, where it is possible to directly capture the geometrical features of each individual layer during a build job using a digital camera. When considering the process of Direct Light Projection (DLP) the possibility of directly capturing the geometrical features of the object during a build job is limited by the specific machine design and the fact that photo-activated monomers often do not change optical characteristics in the polymerization process. Therefore a variant of the previously tested and verified method has been implemented on DLP machine, where instead of capturing the geometrical features of the produced objects during the build job directly these features are captured indirectly by capturing the reflection of the projected light projected during the build job. Test series were made and a reconstruction of two octave spheres were produced and compared with the input CAD file and scans of the produced objects. The comparison showed
\end{abstract}

Ulrik Vølcker Andersen

Department of Mechnical Engineering, Technical University of Denmark, Kgs. Lyngby, Denmark, DK-2800

Tel.: +45-45254833

E-mail: uand@mek.dtu.dk a good correlation between the reconstructions and the scans considering the resolution of the images used for the reconstruction and it was thereby concluded that the method has a promising potential as verification method for DLP machines.

Keywords Additive manufacturing - Tolerance verification · Digitial image processing $\cdot 3 \mathrm{D}$ scanning $\cdot$ Direct Light Projection · Photo-activated polymerization

\section{Introduction}

AM has a unique advantage over conventional production methods in that it has practically no restriction in geometric shape of the products. This allows the AM technologies the potential to be a key component in the mass production of parts with special geometrical requirements. The geometrical freedom, however also poses a challenge when it comes to ensuring a reliable verification of geometrical tolerances. Before the AM technologies can be introduced to the wider range of industries this challenge needs to be met with a high precision verification method that is both cost efficient and simple to implement the production line. Earlier research $[1,2]$ has addressed this challenge by proposing a method for in-line reverse engineering and 3D reconstruction of parts that is additively manufactured on common powder-bed based systems, such as 3DP and SLS. The method has been tested and validated for a 3DP platform $[1,2]$. This paper demonstrates and validates how this method can be adapted to be used with another AM technology namely DLP. Two modified versions of this method have been implemented and tested on an EnvisionTec Perfactory MML [3] machine at the Technical University of Denmark. This article describes the methodology and discusses the issues 
concerning performance raised by these particular implementations.

\section{Method concept}

When developing a common methodology for tolerance verification for all AM technologies it is crucial to identify and exploit the common process similarities. As identified in previous work $[1,2]$ the common process similarity for all AM technologies is that AM methods construct $3 \mathrm{D}$ products by continuously adding thin layer upon layer. This process can be directly translated to the $3 \mathrm{D}$ reconstruction method, where a vision system is used to capture the 2D features of each layer which then are added together to reconstruct a complete $3 \mathrm{D}$ model $[1,2]$. The implementation of this concept requires both a vision system that gathers the raw data in the form of digital images and a numerical algorithm that can convert the raw images into a $3 \mathrm{D}$ reconstruction. The vision system has to be adapted to the specific platform and there might be a need for specific correction of the raw images at a specific machine. The algorithm for producing the $3 \mathrm{D}$ reconstruction and the STL file can however be exactly the same as described in [1] and [2].

\subsection{New implementation}

The concept proposed in this work can be transferred to practically any layer by layer manufacturing process. There are however several different additive manufacturing technologies. These technologies are normally divided into specific categories. Among these categories one of them stands out as the one that is mostly used in industrial manufacturing [5]: Stereolithography Apparatus (SLA). The SLA technology is a process where a fluid medium is altering its physical state at the build platform in response to a controlled stimulant [6]. As SLA is the AM technology with the highest degree of prevalence in the industry, it is of particular interest to prove that the reconstruction method is applicable for this technology. DLP can be seen as a sub category of SLA (where the stimulant is light) and therefore the technology can be seen as a representation of the SLA technologies. In the following it is described how this concept has been fitted to the Perfactory MML.

\section{Method Implementation}

The Perfactory MML is based on a liquid photo-activated monomer, which is formed into a polymer structure

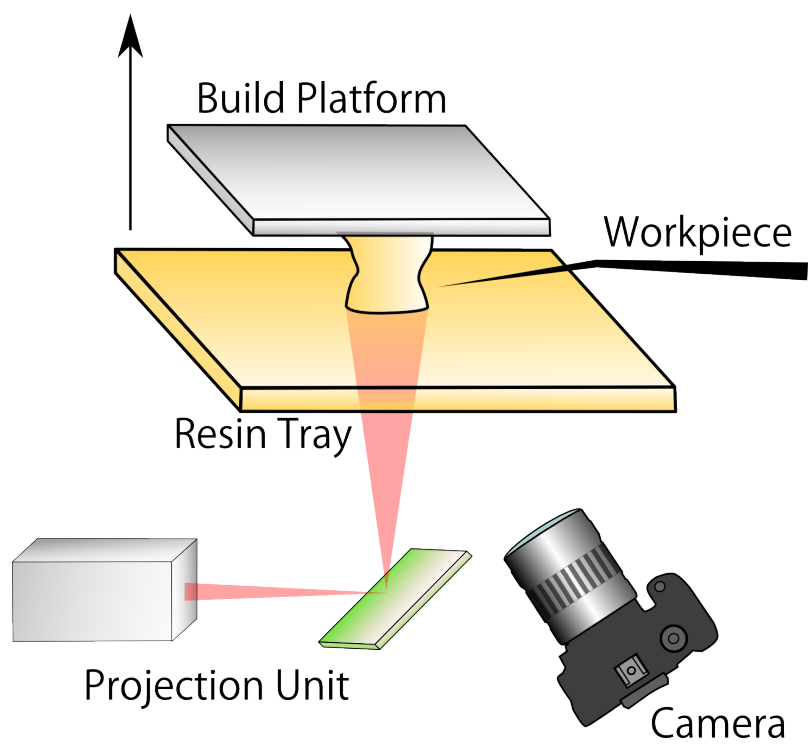

Fig. 1 Sketch of the Perfactory MML. The build platform moves in the direction of the arrow during a build job.

when exposed to UV-light. The monomer is filled into a transparent tray, which is set above a projector. The build platform is a flat surface which can move up and down above the tray. When a build job starts the build platform is moved to the bottom of the tray into the monomer, leaving only a thin layer of monomer between the tray and the build platform. The projector uses a Microelectromechanical system (MEMS) mirror array to expose exactly the desired area on the build platform with UV-light, thus hardening the desired area for the given layer. After each layer has been created the build platform is moved up one layer. A mechanical mechanism tilts the tray to let the monomer float under the newly formed polymer structure and back to a fully horizontal position and then the next layer can be created and so forth. In this way the desired design is "drawn" up of the polymer liquid up-side-down as shown on figure 1.

Considering all possible configurations of the machine the XY resolution is between 16 and $60 \mu \mathrm{m}$ and the $\mathrm{Z}$ resolution (how much the build platform moves between each layer) is between 15 and $150 \mu \mathrm{m}$ [3]. From experience we know that the build platform is exposed to light from two intervals for each layer. The two intervals last for about 4-5 seconds each and are separated from each other with a times of less than 10 seconds. The two intervals are related to the Enhanced Resolution Module (ERM), that consist of a $\frac{1}{2}$ pixel shift in the $\mathrm{X}$ - and $\mathrm{Y}$ - directions, which is supposedly create the effect of a doubled resolution [3]. 


\subsection{The vision acquisition system}

The liquid monomer and the solid polymer have the same color, therefore in the vision acquisition system for the Perfactory MML it is chosen to look at the reflection of the UV-light instead of the actual object that is being built. The vision acquisition system used for the Envision Tec was based on a single-lens reflex (SLR) digital camera ${ }^{1}$ which is placed next to the projector looking up towards the tray. The light from the projector is reflected at the bottom of the tray downward to the camera. This gives an image showing where the hardening process takes place. During the build job the camera is connected to a computer from which it is controlled. Sharp images are achieved by letting the camera run in full manual mode (to avoid compensation for a low amount of light) and by using low shutter. From the computer the camera is triggered. Various system to ensure the camera is triggered at the correct time (that is when the build platform is exposed to light) have been tested, but in the end it was found that the most efficient method was to simply take pictures with a short enough interval to be sure that all layers are captures. In the test series described in section 5 a sample rate of about $0.8 \mathrm{~Hz}$ was used.

\subsection{Post-processing of 2D images}

The projector unit is placed directly under the build platform and it is therefore impossible to also put the camera directly under the build platform. Instead the camera is placed beside the projector unit with a tilt angle, so that the camera is still focusing on the build platform ${ }^{2}$. The tilt angle however introduces a deformation of the image due to the perspective as seen on figure 2. The deformation means the angles are twisted so that the square becomes a none-uniform quadrilateral. Two different methods to overcome this problem were identified: One solution is to introduce a geometrical consideration of how the images are deformed due to the tilt angle and thereby create a reverse deformation model that would precisely remove the deformation from the tilting. Another solution is to use already existing photo manipulation software to recreate the right angled corners of the objects thus creating rectangular images of the figures. In this work the latter solution was applied for the presented results. For this the open

\footnotetext{
1 Canon EOS 1000D with 18-55mm stock lens. The camera has a resolution of $3888 \times 2592$.

2 It was not possible to limit the focus to the build platform, in fact a large proportion of the images capture an area without interest for reconstruction.
}

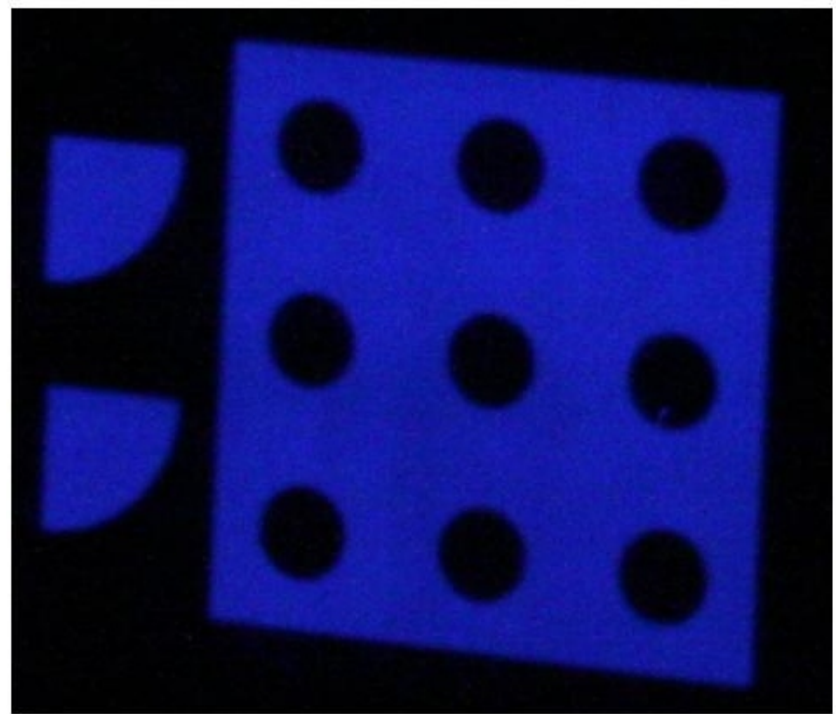

Fig. 2 Example of a raw image from the test (the test is described in section 4). The image is cut around the figures and consists of $470 \times 405$ pixels.

source image manipulation program GIMP $2.0^{3}$ was used. For the treatment described in the following a script for Matlab is used.

When the images have been reshaped they will be changed into black and white (binary) images where the white areas represent the areas where something has been build and the black where nothing has been build. This process is done using a simple threshold function, where the color (RGB) value of each pixel is examined and compared with a threshold. The pixel with a higher value becomes white and the pixel with a lower value becomes black. It was noticed during the experiments that it was sufficient to use the blue pixel value for the thresholding and this was therefore implemented in the final algorithm. When all images have been treated this information is then used to produce the $3 \mathrm{D}$ reconstruction.

\section{Experimental}

In order to validate the verification system on the Perfactory MML, a production series has been carried out. In this series, three objects have been created in a single build job, with the camera taking images as described in section 3 . Using one of the objects to calibrate, reconstructions of the other two objects are realized.

The images were sorted by layers, added together for each layer and cut out around the build platform. The resulting images were then reshaped using GIMP. On

3 GIMP: GNU Image Manipulation Program, an open source image manipulation program 


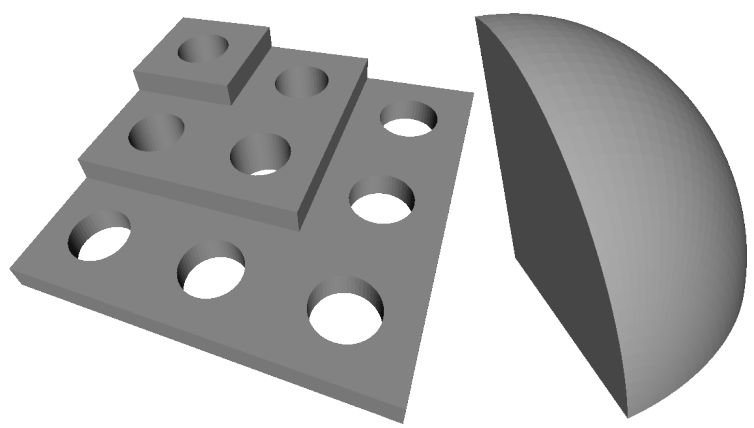

Fig. 3 Plate with holes and an octave sphere

the reshaped images length measurement were performed for one of the objects and compared with the $2 \mathrm{D}$ measurement of the real object. This comparison leads to the calibration. Using this calibration, the reshaped images and the Matlab script described in section 3, STL files of each of the three objects were created. The three objects were also scanned, so that a threefold comparison could be made: the 3D-scan vs. the input STL files, the input STL files vs. the reconstructed STL files and the 3D-scan vs. the reconstructed STL files.

\subsection{Test objects}

When choosing the objects for the tests it was important to have different geometrical features in order to test how well these features can be measured. It was important to have some easily identifiable lengths which could be used for the calibration. It was also important to have some true $3 \mathrm{D}$ shapes, which have a gradual change in the shape for each layer, in order to verify whether such features can be measured correctly. Another important consideration is the duration of the build job. This is important due to the relative large amount of data produced (each image is about 2.3 MB, with a sampling rate of $0.8 \mathrm{~Hz}$ that is about $1.8 \mathrm{MB} / \mathrm{s}$ ). For these reasons two different objects with a relatively small size and a low z-resolution to reduce the number of layers were chosen. The objects are a step-plate with holes and two octave spheres.

\subsection{Calibration}

Geometry measurements enable the calculation of the calibration factor between lengths at the images and lengths of the objects, which is needed to provide a scaled reconstruction. The calibration factor is calculated as

$$
\begin{aligned}
& s f(1)=\operatorname{Li}(1) / \operatorname{Lm}(1) \\
& s f(2)=\operatorname{Li}(2) / \operatorname{Lm}(2)
\end{aligned}
$$

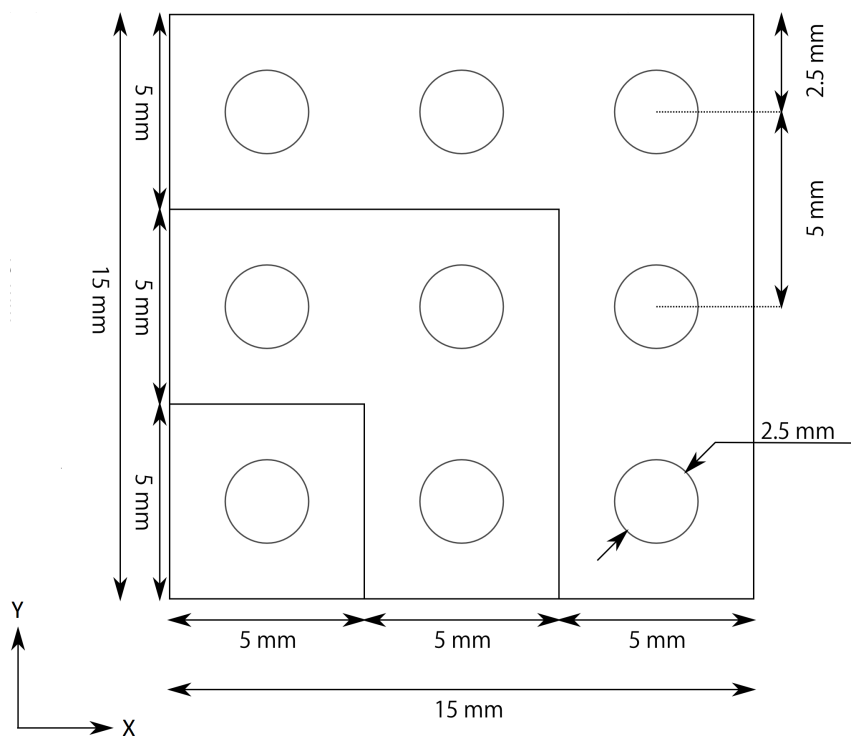

Fig. 4 The dimension of the plate in the plane in the input file. The height of each step is $1.125 \mathrm{~mm}$, giving a total height of $3.375 \mathrm{~mm}$.

where sf is the calibration factor in pixel per $\mathrm{mm}, \mathrm{Li}$ is any length on one of the images in pixel and $\mathrm{Lm}$ is the corresponding length measured on the real object in mm. Index 1 indicates it is the calibration factor or length in the $\mathrm{X}$-direction and index 2 the Y-direction with the $\mathrm{X}$ - and Y-directions defined as in figure 4 .

The calibration measurements were performed at a Carl Zeiss 3D coordinate measuring machine of type OMC 850 [4]. The coordinate system of the measurements has its origin at the bottom left corner of the plate (as seen in figure 5). The $\mathrm{X}$ - is parallel to the bottom edge of the plate and the XY-plane is fitted to lower step on the plane (the step containing the circles $3,6,7,8$ and 9 as defined on figure 5). Each of the 9 cylindrical holes were defined and measured as circles, given the radius and the coordinates of the center for each circle. The coordinates of the circle center points (CCPs) allowed us to calculate up to 36 different distances that can be compared with the images. The measurements were performed 6 times without moving the plate between the measurements. As it can be seen from figure 6 and table 1, there are some differences between the measured and the nominal CCPs, while the differences between the individual measurements are smaller. This indicates the produced plate deviates from the model.

The distances along the $\mathrm{X}$ - and Y-direction between the CCPs were calculated useing each set of measurements separately.

From table 2 and 3 it can be seen that the standard deviation of the distance measurements is up to $2.1 \mu \mathrm{m}$, which is within the accuracy of the coordinate measuring machine [4]. In order to give an overall es- 


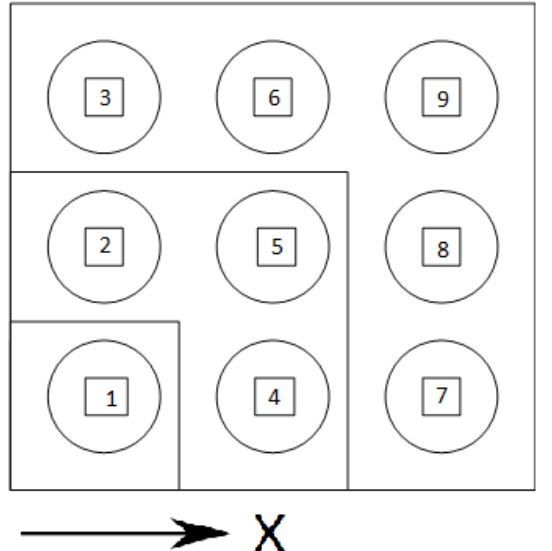

Fig. 5 Sketch of measured object, with the definition of the numbering and the $\mathrm{X}$-axis

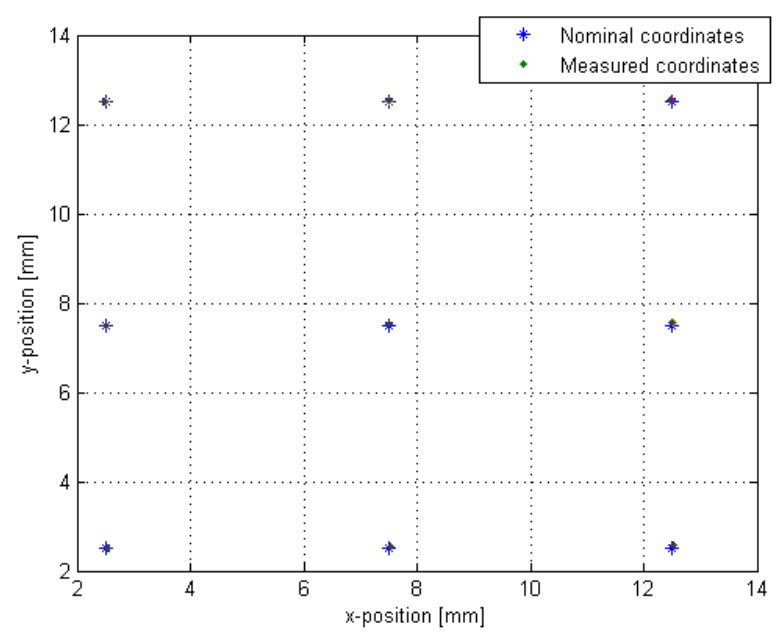

Fig. 6 Nominal and measured circle center points. The coordinates of the nominal points are the coordinates of the circle center points as define in figure 4 .

Table 1 Distance from measurements to nominal points of the circle center points in $\mu \mathrm{m}$.

\begin{tabular}{lrrrrrr}
\hline & M 1 & M 2 & M 3 & M 4 & M 5 & M 6 \\
\hline Circle1 & 33.7 & 36.5 & 35.4 & 35.8 & 34.9 & 34.7 \\
Circle2 & 15.2 & 16.2 & 16.0 & 17.0 & 16.5 & 15.8 \\
Circle3 & 7.0 & 9.3 & 8.1 & 7.3 & 6.8 & 7.1 \\
Circle4 & 45.3 & 48.5 & 47.6 & 47.2 & 46.5 & 47.4 \\
Circle5 & 21.4 & 23.4 & 22.0 & 22.9 & 22.0 & 22.4 \\
Circle6 & 27.0 & 28.9 & 27.6 & 27.7 & 26.6 & 27.5 \\
Circle7 & 68.1 & 71.1 & 69.7 & 69.4 & 68.1 & 68.9 \\
Circle8 & 54.6 & 58.1 & 56.3 & 56.1 & 54.8 & 55.3 \\
Circle9 & 52.4 & 54.4 & 55.3 & 53.7 & 52.6 & 52.5 \\
\hline
\end{tabular}

Table 2 Mean values, standard deviation and ratio between standard deviation and mean values of measurements of Xdistances between the circle center points.

\begin{tabular}{|c|c|c|c|}
\hline Distance from & Mean $[\mathrm{mm}]$ & $\mathrm{STD}[\mu \mathrm{m}]$ & $\frac{S T D}{\text { mean }} \times 10^{4}$ \\
\hline Circle 1 to circle 4 & 5.0017 & 0.8 & 1.59 \\
\hline Circle 1 to circle 5 & 4.9746 & 0.9 & 1.84 \\
\hline Circle 1 to circle 6 & 4.9618 & 1.2 & 2.34 \\
\hline Circle 1 to circle 7 & 9.9862 & 0.3 & 0.26 \\
\hline Circle 1 to circle 8 & 9.9774 & 0.8 & 0.76 \\
\hline Circle 1 to circle 9 & 9.9485 & 1.1 & 1.10 \\
\hline Circle 2 to circle 4 & 5.0190 & 1.2 & 2.35 \\
\hline Circle 2 to circle 5 & 4.9918 & 0.6 & 1.23 \\
\hline Circle 2 to circle 6 & 4.9791 & 0.8 & 1.51 \\
\hline Circle 2 to circle 7 & 10.0035 & 0.4 & 0.39 \\
\hline Circle 2 to circle 8 & 9.9947 & 0.4 & 0.41 \\
\hline Circle 2 to circle 9 & 9.9657 & 0.7 & 0.75 \\
\hline Circle 3 to circle 4 & 5.0388 & 2.1 & 4.21 \\
\hline Circle 3 to circle 5 & 5.0117 & 0.7 & 1.40 \\
\hline Circle 3 to circle 6 & 4.9989 & 0.6 & 1.23 \\
\hline Circle 3 to circle 7 & 10.0234 & 1.4 & 1.36 \\
\hline Circle 3 to circle 8 & 10.0146 & 0.9 & 0.88 \\
\hline Circle 3 to circle 9 & 9.9856 & 0.7 & 0.71 \\
\hline Circle 4 to circle 7 & 4.9845 & 0.9 & 1.76 \\
\hline Circle 4 to circle 8 & 4.9757 & 1.3 & 2.64 \\
\hline Circle 4 to circle 9 & 4.9468 & 1.7 & 3.45 \\
\hline Circle 5 to circle 7 & 5.0116 & 0.9 & 1.83 \\
\hline Circle 5 to circle 8 & 5.0028 & 0.6 & 1.18 \\
\hline Circle 5 to circle 9 & 4.9739 & 0.7 & 1.41 \\
\hline Circle 6 to circle 7 & 5.0244 & 1.0 & 2.00 \\
\hline Circle 6 to circle 8 & 5.0156 & 0.5 & 0.89 \\
\hline Circle 6 to circle 9 & 4.9867 & 0.3 & 0.67 \\
\hline
\end{tabular}

timation of the uncertainty of the measurements the standard deviation divided by the mean value is calculated for each distance, as seen in table 2 and 3 . The uncertainty of each measurement was estimated based on the standard deviation:

$U_{i}=2 \frac{D_{i, S T D}}{D_{i, \text { mean }}}$

And considering the uncertainty each distance can be written as:

$D_{i}=D_{i, \text { mean }} \pm U_{i} D_{i, \text { mean }}$

Or if considering one uncertainty for all the measurements:

$D_{i}=D_{i, \text { mean }} \pm U D_{i, \text { mean }}$

Where this uncertainty can be assumed to be:

$U=\max \left(U_{i}\right)$

By doing this the uncertainty for distances in X-direction is $4.21 \times 10^{-1} \frac{\mu m}{m m}$ and for distances in Y-direction it is $2.59 \times 10^{-1} \frac{\mu m}{m m}$. In order to calculate the calibration factor the corresponding distances on the images in pixels have to be calculated. For this calculation only the images of the lower 22 layers are used, as the upper layers does not include all 9 holes. The center points of the 
Table 3 Mean values, standard deviation and ratio between standard deviation and mean values of measurements of Ydistances between the circle center points.

\begin{tabular}{lrcc}
\hline Distance from & Mean $[\mathrm{mm}]$ & STD $[\mu \mathrm{m}]$ & $\frac{S T D}{\text { mean }} \times 10^{4}$ \\
\hline Circle 1 to circle 2 & 4.9919 & 0.8 & 1.56 \\
Circle 1 to circle 3 & 9.9925 & 0.8 & 0.80 \\
Circle 1 to circle 5 & 5.0078 & 0.6 & 1.13 \\
Circle 1 to circle 6 & 10.0135 & 0.6 & 0.61 \\
Circle 1 to circle 8 & 5.0416 & 0.5 & 1.05 \\
Circle 1 to circle 9 & 10.0366 & 1.1 & 1.12 \\
Circle 2 to circle 3 & 5.0007 & 0.4 & 0.72 \\
Circle 2 to circle 4 & 4.9729 & 1.0 & 1.92 \\
Circle 2 to circle 6 & 5.0217 & 0.5 & 0.90 \\
Circle 2 to circle 7 & 4.9386 & 0.9 & 1.81 \\
Circle 2 to circle 9 & 5.0447 & 1.0 & 1.88 \\
Circle 3 to circle 4 & 9.9736 & 0.9 & 0.94 \\
Circle 3 to circle 5 & 4.9848 & 0.5 & 1.03 \\
Circle 3 to circle 7 & 9.9393 & 0.8 & 0.81 \\
Circle 3 to circle 8 & 4.9510 & 1.0 & 2.04 \\
Circle 4 to circle 5 & 4.9888 & 0.8 & 1.52 \\
Circle 4 to circle 6 & 9.9946 & 0.9 & 0.86 \\
Circle 4 to circle 8 & 5.0226 & 1.3 & 2.59 \\
Circle 4 to circle 9 & 10.0176 & 1.8 & 1.76 \\
Circle 5 to circle 6 & 5.0058 & 0.2 & 0.49 \\
Circle 5 to circle 7 & 4.9545 & 0.5 & 1.05 \\
Circle 5 to circle 9 & 5.0288 & 1.2 & 2.47 \\
Circle 6 to circle 7 & 9.9603 & 0.5 & 0.51 \\
Circle 6 to circle 8 & 4.9720 & 0.7 & 1.47 \\
Circle 7 to circle 8 & 4.9883 & 0.3 & 0.66 \\
Circle 7 to circle 9 & 9.9833 & 1.1 & 1.11 \\
Circle 8 to circle 9 & 4.9950 & 1.2 & 2.39 \\
\hline
\end{tabular}

holes are found using the part of the $3 \mathrm{D}$ reconstruction algorithm that produces a boundary map from each image. From the boundary map the position of the CCPs are found as the mean of the extreme coordinates of the boundary points around the circles.

Let $\mathrm{X}$ be the $\mathrm{X}$-coordinates of the boundary point around the circle and $\mathrm{Y}$ the $\mathrm{y}$-coordinates, then the extreme $\mathrm{x}$ coordinates $\left(x_{1}\right.$ and $\left.x_{2}\right)$ and the $\mathrm{x}$-coordinate of the $\mathrm{CCP}(\mathrm{xc})$ is calculated as:

$x_{1}=\min (X)$

$x_{2}=\max (X)$

$x_{c}=\frac{x_{1}+x_{2}}{2}$

Similar the y-coordinates are calculated:

$y_{1}=\min (Y)$

$y_{2}=\max (Y)$

$y_{c}=\frac{y_{1}+y_{2}}{2}$

For each of the images all the distances between the CCPs are found in number of pixel in $\mathrm{X}$ - and $\mathrm{Y}$ direction separately. The ratio between the distances

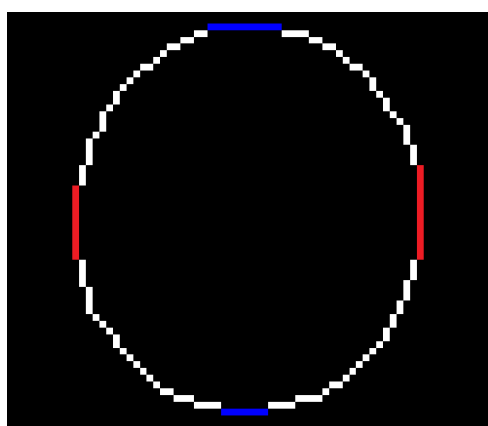

Fig. 7 Example of calculating the center point of a circle. The red color marks where the extreme X-coordinates are found, the blue color marks where the extreme Y-coordinates are found. The extreme X-coordinates are here 264 and 315 and the extreme Y-coordinates are 83 and 140, therefore the center point is estimate to be at $(289.5,111.5)$

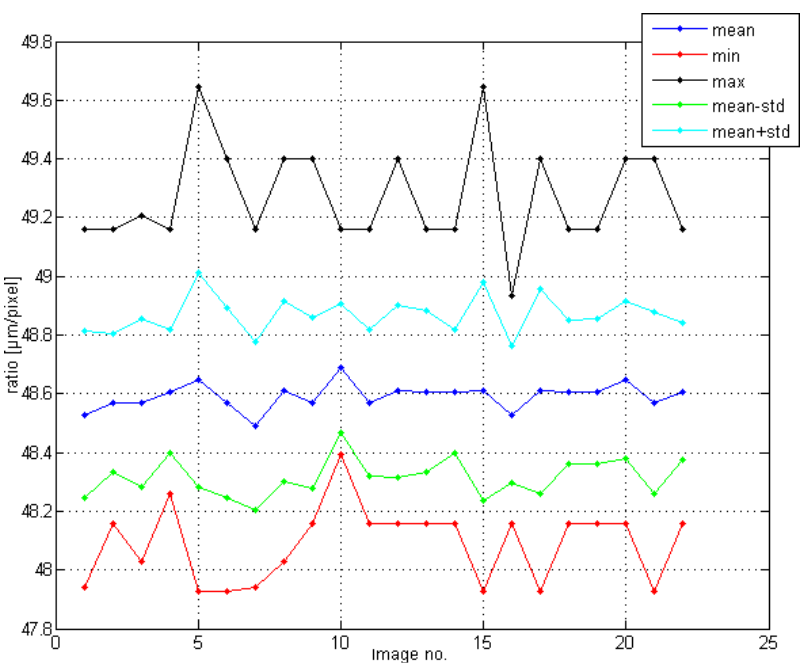

Fig. 8 mean, min, max and mean $+/$ - standard deviation of the ratio between the distances in the X-direction in $\mathrm{mm}$ and in pixel.

obtained from the measurements and those obtained from the images are calculated for X- and Y-direction separately for those instances where the distance is more than $1 \mathrm{~mm}$.

For each image a total of 27 ratios between the $\mathrm{X}$ distance in pixel and the corresponding distance in $\mathrm{mm}$ have been calculated and another 27 for the Y-distances. The overall mean values of the calculated ratios are $48.59 \mu \mathrm{m} /$ pixel for the X-axis and $44.00 \mu \mathrm{m} /$ pixel for the Y-axis.

The uncertainty of the image measurements on the images can be estimated the same way as for the surface measurements. This gives an uncertainty of $8.06 \frac{\mu \mathrm{m}}{\mathrm{mm}}$ for the X-direction and $7.62 \frac{\mu m}{\mathrm{~mm}}$ for the Y-direction. Combining the uncertainties of image measurements and the surface measurements the total uncertainty of the ratio calculation is $8.48 \frac{\mu \mathrm{m}}{\mathrm{mm}}$ for the X-direction and $7.88 \frac{\mathrm{\mu m}}{\mathrm{mm}}$ for the Y-direction. This means that the calibrations 


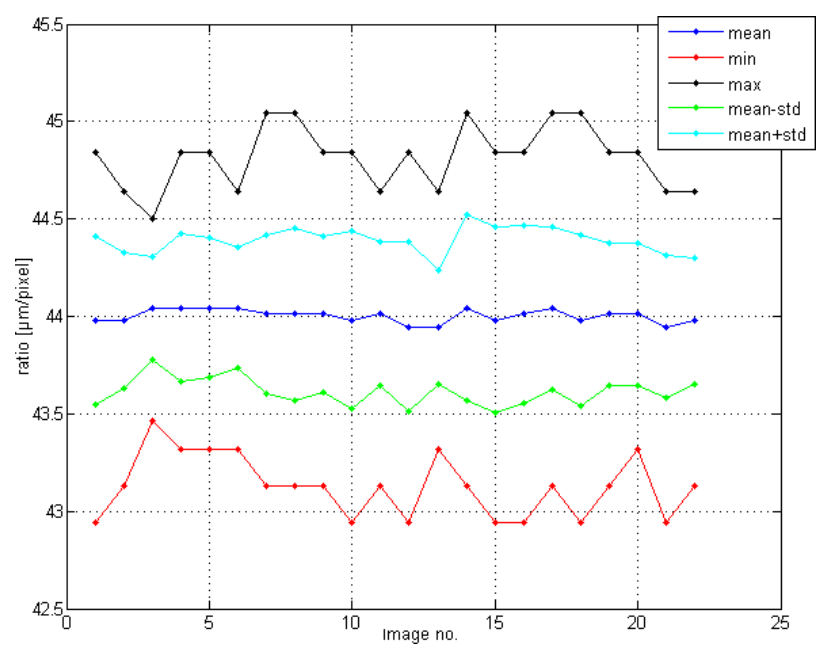

Fig. 9 mean, min, max and mean $+/$ - standard deviation of the ratio between the distances in the Y-direction in $\mathrm{mm}$ and in pixel.

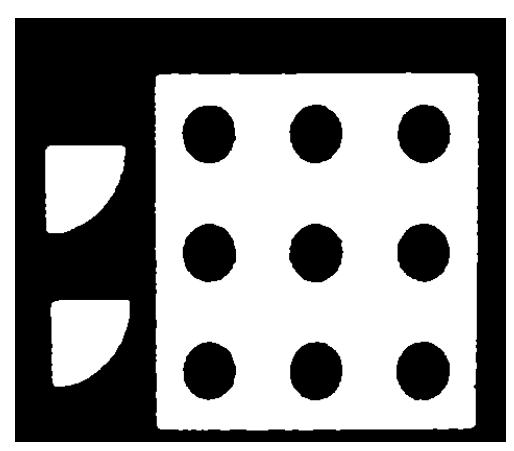

Fig. 10 Example of a black and white image that contains the geometry of one layer

\section{factors are:}

Calibration factor X-direction

$=48.6 \mu \mathrm{m} /$ pixel $\pm 0.4 \mu \mathrm{m} /$ pixel

Calibration factor $\mathrm{Y}$-direction

$=44.0 \mu \mathrm{m} / \mathrm{pixel} \pm 0.4 \mu \mathrm{m} / \mathrm{pixel}$

\subsection{D-reconstruction and comparison}

The images of each layer of the build job and the calibration factors contain all the information needed to make a 3D-reconstruction of the build job. The images are transformed into black and white images, one example is shown in figure 10. The images have been cut into 3 pieces, one for the square with holes and one for each of the two octave spheres. The cut images have then been stacked together to create three separate 3D- reconstruction as STL-files. The flat sides and the round side of the reconstruction do not appear very smooth. This is due to the fact the boundaries of the objects lay

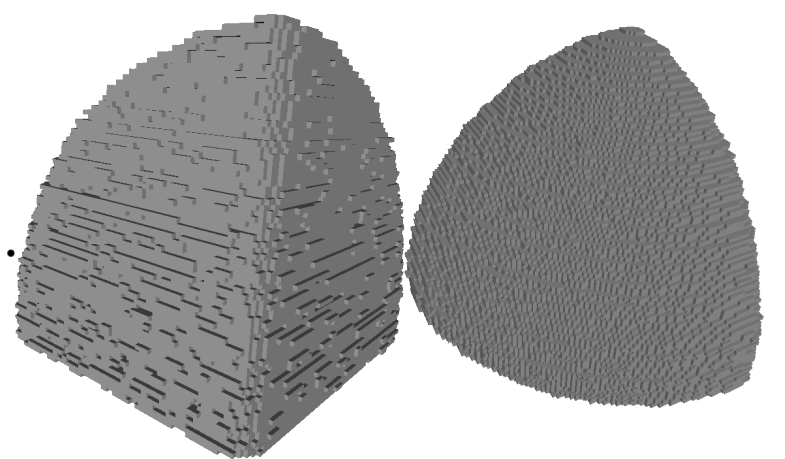

Fig. $113 \mathrm{D}$ reconstrution of octave sphere 1 seen from the flat side and seen for the round side

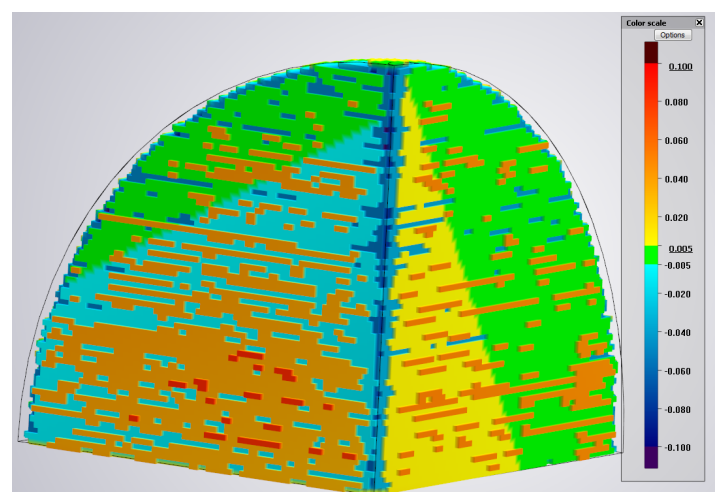

Fig. 12 Comparison of reconstruction with CAD file for octave sphere 1 . View from the flat side

at one pixel and not between the pixels, whereas the reconstruction program will decide whether a pixel on the boundary is part of the object or not. This decision will differ from pixel to pixel and from layer to layer in a manner that will appear to be random whereas the reconstructed objects will end up with this dotted surface. The size of these "dots" is the size of the pixels in the image and the layer height in z-direction. Therefore the reconstruction can never be more accurate than the resolution of the images taken during the build job.

The reconstructions have been compared to the input CAD file using the program Convince from 3shape. Here the CAD file has been used as reference and the reconstruction have been turned and moved to achieve the best possible fit using an built-in algorithm of Convince. Comparing the reconstruction and the input file it is found that the maximum difference is $0.1284 \mathrm{~mm}$. From figure 12 and 13 it can be seen that the objects tends to be a bit too small at the edges and too large at the center of the surfaces. The comparison does not tell how much of the error comes from the actual production or from the reconstruction. Instead a 3D scan of each of the two produced octave spheres has been made on a 3shape Q700 3D scanner [8].

The scans have been compared with reconstructions us- 


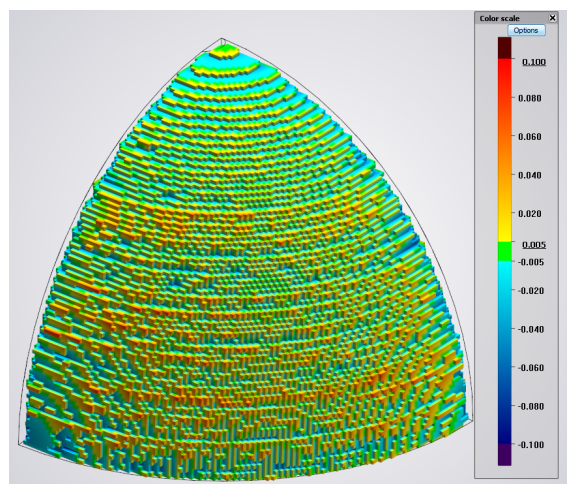

Fig. 13 Comparison of reconstruction with CAD file for octave sphere 1 . View from the round side
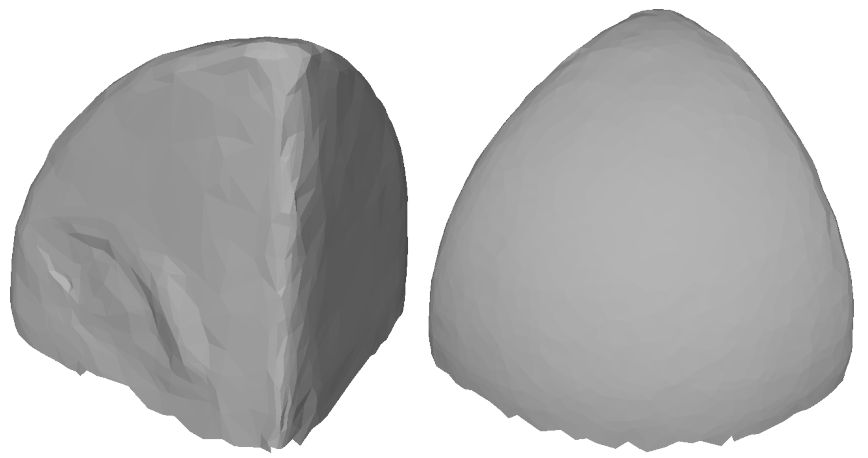

Fig. 14 Scan of octave sphere 1

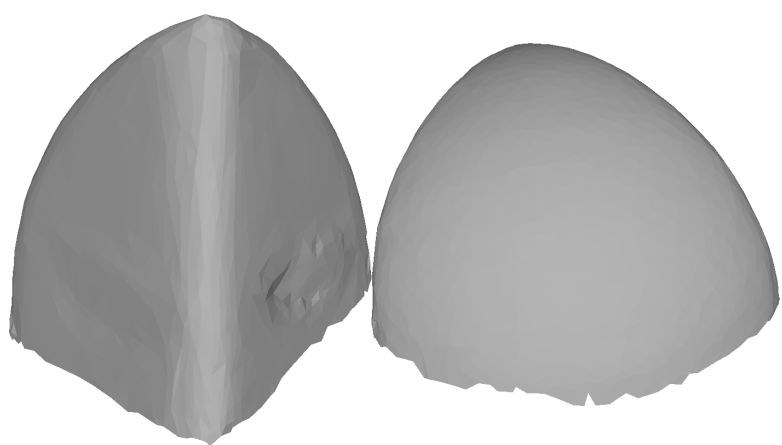

Fig. 15 Scan of octave sphere 2

ing the program Convince. Since the scans do not include the bottom of the objects they are not suitable to be used as references in the comparisons. Instead the reconstructions are used as references in the comparisons between the scans and the reconstructions, meaning that the difference between the object sizes seen in the comparison is how much the scans are larger than the reconstructions. The comparison between the scans and the reconstructions shows that there is considerably less difference between the scans and the reconstruction than between the input file and the reconstructions. There is however some difference, the maximum is 79.3 $\mu \mathrm{m}$.

These values gives an idea of how good the presented

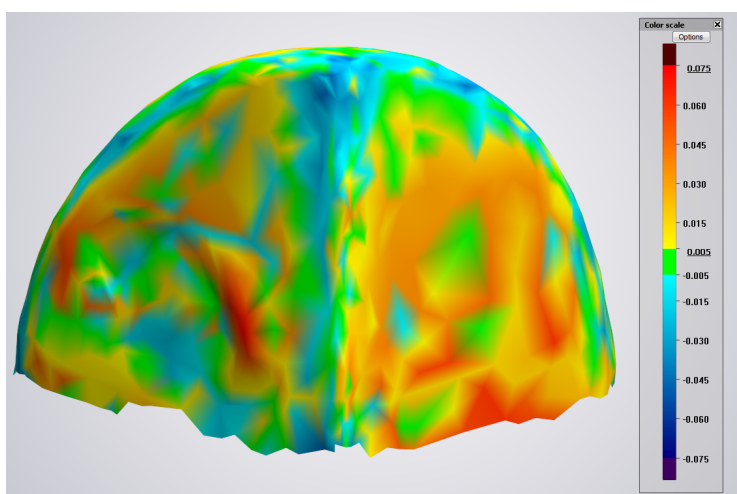

Fig. 16 Comparison between scan and reconstruction of octave sphere 1 , see from the flat side

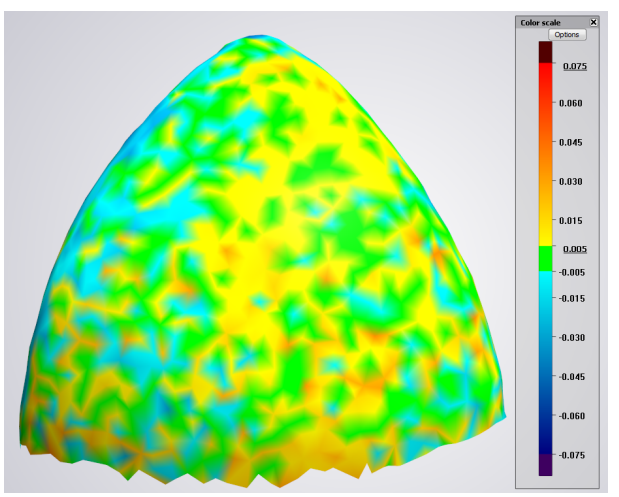

Fig. 17 Comparison between scan and reconstruction of octave sphere 1 , see from the round side

reconstruction method is, but it is necessary to bear in mind that the deviation between the scan and the reconstruction can come from three different sources; deformations in the object during or after the build job, the reconstruction procedure and the scan. Since each source have not been quantified individually it has not been established how much of the deviation derives from inaccuracies in the reconstruction and how much derives from deformations and inaccuracies in the scan.

\section{Discussion}

The verification system was implemented in Perfactory MML machine and tested for a build job containing three objects. One object was used for calibration and the two others were used for reconstruction to determine the quality of the reconstruction method. The results showed that the maximum difference between the reconstruction and the scanning of the produced objects was $79.3 \mu \mathrm{m}$. This proves a very reasonable correlation especially when considering the many uncertainties involved in the reconstruction. The source of the deviation is threefold: deformations in the object after polymerization, inaccuracies in the scan and inaccura- 
cies in the reconstruction. The level of deformations in the objects has not been investigated in this work, but it is possible that there are some deformations if the material either shrink or expand during the hardening process. From the documentation of the 3shape scanner it is known that the inaccuracies of the scans are up to $16 \mu \mathrm{m}[8]$.

The accuracy of the reconstruction procedure can be split in to three parts:

a) The images

b) The numerical treatment of the images

c) The calibration

The accuracy of the images depends on how well the images reproduce the actual build job. With the Perfactory MML one key concern is how well the reflected light represents what is actually happening in the build job. In the reconstruction procedure it is assumed that the area of the reflected light at the bottom of the tray corresponds exactly to the area of the build job, any deviation from that fact is a source of error. Another factor relevant to the accuracy of the images is the quality of the camera, especially the resolution. The reconstruction can never be better than the resolution of the images. Since one pixel represent either a part of the object or not, the accuracy of the reconstruction cannot be less than one pixel. The pixel size of the build job was $42 \mu \mathrm{m}$ in the XY-plane [3] and therefore smaller than the pixel size of the images. In order to have a proper reconstruction of build job the resolution of the images should be significant larger than the resolution of the build job. It is therefore necessary to have a much higher resolution of images. If the resolution of the images were 10 times higher than the resolution of the build job (corresponding to a pixel sizes in the images of about $4 \mu \mathrm{m}$ ) the resolution of the images would be $6250 \times 5000$ pixels (32.5 mega pixels), since the size of the build was about $25 \times 20 \mathrm{~mm}$.

The numerical treatment of the images may introduce errors if a wrong threshold is used when changing the images into black and white images. One challenge in finding a correct threshold value is the fact that the light intensity differs from image to image. However from the experience of the presented work it is noted that the images produced in this work have a clear distinction between colored and non-colored pixel values, whereas minor changes in the threshold value only effects the pixels at the borderline between lighted area and not lighted area. This means that the size of the error introduced via the thresholding of the images is in the order of one pixel around the edges of the objects, and therefore a higher resolution would reduce this error.

\section{Conclusions}

This article expands on the 3D reconstruction method presented in [1] and [2] to be used in an DLP machine, where the method is tested and validated on a build job of a step-plate with holes and two octave spheres. The $3 \mathrm{D}$ reconstruction of the octave sphere shows deviation in comparison to 3D scans of about $80 \mu \mathrm{m}$, which indicate the strong potential of the method. Although this deviation is relatively large it is believed that the reconstruction method has the potential to become very accurate if high resolution images are obtained, it is however neccesary to conduct more tests in order to verify the accuracy of the use of indirect measurements in a DLP build job.

\section{References}

1. Pedersen D B et al., In-line monitoring and reverse 3D model reconstruction in additive manufacturing, The 7 th international workshop on microfactories, Daejeon Korea, 24-27 oct. 2010

2. Hansen $\mathrm{H} \mathrm{N}$ et al., Absolute 3D geometry reconstruction of complex additive manufactured parts using layered mesh generationabsolute 3D geometry reconstruction of complex additive manufactured parts using layered mesh generation, 16th European Forum on Rapid Prototyping and Manufacturing, Paris France, 23-24 june 2011

3. Perfactory@3 SXGA+ W/ERM Mini Multi Lens, envisionTEC GmbH

4. Hjelmbjerg F, 3D coordinate measuring machine Calibration Certificate no. S017156, june 2006

5. Terry Wohlers, Wohlers report 2007, State of the Industry Annual Worldwide Progress Report, 2007

6. Charles W. Hull, Apparatus for production of threedimensional objects by stereolithographic, United State Patent number 4,575,330 (1986)

7. Tony Brown, Perfactory Buyers' Guide, Envisiontec UK, Trent UK (2006)

8. 3shape (2011) 3Shape Convince 3D Scanning and Quality Control www.3dscanco.com/pdfs/3shape.pdf 